\title{
Mutual Coupling Compensation in Uniform Circular Arrays with Center Element using a Coupling Matrix based on Phase Modes
}

\author{
Hendrik Rogier* \\ *Information Technology Department, Ghent University \\ St.-Pietersnieuwstraat 41, B-9000 Ghent, Belgium. \\ E-mail: hendrik.rogier@intec.UGent.be, \\ Tel.: ++32/92643343 Fax: $++32 / 92649969$.
}

\begin{abstract}
By expanding the radiation characteristics into phase modes, a coupling matrix is constructed to compensate for mutual coupling in uniform circular arrays with a center element. Relying on a limited number of phase modes, this matrix fully describes the dependency of the receive and transmit characteristics on azimuth angle, for a given elevation angle and for arbitrary terminations placed at the different antenne ports. A sample dual band uniform circular array with center element illustrates the use of the coupling matrix for direction of arrival estimation.
\end{abstract}

\section{INTRODUCTION}

Mutual coupling between elements in antenna arrays has an important impact on the communication characteristics of a wireless link. In an array configuration, the EM characteristics of the antenna elements differ from the properties of the stand-alone elements because of voltages induced by terminal currents in the loads of neighboring elements, described by an impedance matrix $\overline{\bar{Z}}$, and by deformation of the radiation pattern of the stand-alone antenna elements due to currents induced in the open-circuited neighboring antenna elements (shadowing effects) or neighboring scatterers (platform effects). To describe this second phenomenon, the concept of impedance matrix $\overline{\bar{Z}}$ was generalized to a coupling matrix $\overline{\bar{C}}$, and direction-of-arrival (DOA) estimation algorithms such as e.g. the multiple signal classification (MUSIC) algorithm were modified to account for mutual coupling. Uniform circular arrays (UCAs) provide a $360^{\circ}$ scan angle and a beam-width which is nearly independent from the scan angle. However, mutual coupling effects are generally more severe in UCAs, as shadowing and platform effects play a larger role than in the linear configuration. In [1], a coupling matrix was constructed based on a limited number of phase-modes/spherical-modes to describe mutual coupling in UCAs.

In this contribution, we extend the model in [1] by constructing a coupling matrix for UCAs with an additional antenna element [2-4], placed in the center of the array. Given the phase-mode description for the UCA with center element in Section II, the coupling matrix is constructed in Section III. In [4], the coupling matrix described the radiation characteristics in the azimuth plane. Here a general, but fixed elevation angle is considered. The use of a coupling matrix for mutual coupling compensation in direction-of arrival (DOA) estimation is illustrated in Section IV.

\section{Phase-mode Description of The UCA with CENTER ELEMENT}

Consider a UCA consisting of $N$ elements: $N-1$ identical antennas uniformly distributed on a ring with radius $r$ and with one antenna in the center of the ring, as in Fig. 2. The center element, with open-circuit voltage $V_{0,1}(\theta, \phi)=V_{0,1}\left(\theta, \phi-\frac{2 \pi}{N-1}\right)$, does not need to be identical to the antennas on the ring, for which the open-circuit voltage is given by $V_{0, i}(\theta, \phi)=V_{0,2}(\theta, \phi-$

H. Rogier is a Postdoctoral Fellow of the FWO-V. His research is supported by a grant of the DWTC/SSTC, MOTION project. 
$\left.(i-2) \frac{2 \pi}{N-1}\right)$, for $i=2, \ldots, N$. The input impedance matrix of the array is described by $\overline{\bar{Z}}=$ $\left(\begin{array}{c|c}Z_{1,1} & Z_{1,2} \mathbf{I}_{1 \times(N-1)} \\ \hline Z_{1,2} \mathbf{I}_{(N-1) \times 1} & \overline{\bar{Z}}_{\text {outer }}\end{array}\right)$, where $\overline{\bar{Z}}_{\text {outer }}$ has a Toeplitz structure.

We now decompose both the open-circuit voltages and the matrix $\overline{\bar{Z}}$ into phase modes. In [1], it is shown that, for each antenne element $i$, the decomposition of the open-circuit voltage $V_{0, i}(\theta, \phi)=$ $\sum_{m=-M}^{+M} V_{0, i, m}^{\phi}(\theta) e^{j m \phi}$ can be restricted to $m \in[-M, M]$, provided that $M \gg \frac{2 \pi d}{\lambda}$, with $d$ the largest dimension of the array and $\lambda$ the wavelength. Choosing $M=\left\lceil 4 \frac{d}{\lambda}+4\right\rceil$ yields an accuracy better than $0.1 \%$. As in [1], for each phase mode $m$ the matrix $\overline{\bar{Z}}_{\text {outer }}$ reduces to the phasesequence impedance $Z_{\text {outer }, m}^{\phi}=\sum_{n=1}^{N-1} Z_{\text {outer }, i-1, n} e^{-j \frac{2 \pi m(i-1-n)}{N-1}}$. Because of symmetry, this impedance is independent of the port number $i=2, \ldots, N$. Because of its location and rotational symmetry, the inner antenna element 1 only gives rise to phase modes of the orders $l(N-1)(l=$ $0,1, \ldots)$. For these modes, the circuit model shown in Fig. 1 is described by the impedance $Z_{1,1}$, the phase-mode open-circuit voltage $V_{0,1, l(N-1)}^{\phi}(\theta)$ and a voltage source describing the coupling to the outer antenna elements, given by $(N-1) Z_{1,2} I_{2, l(N-1)}^{\phi}$, with $I_{2, l(N-1)}^{\phi}$ the phase-mode current for the outer antennas. The phase-mode current on the center antenna $I_{1, l(N-1)}^{\phi}$ contributes to the phase-mode voltage of the outer antennas under the form of a voltage source $Z_{1,2} I_{1, l(N-1)}^{\phi}$. For all other orders of phase modes, the outer elements do not interact with the center element and the circuit model derived in [1] still holds, i.e. the outer-port circuit in Fig. 1 does not couple to the inner element (no inner-port circuit and no contribution of $Z_{1,2}$ ). The phase-mode currents and voltages at the different ports are uncoupled for different mode orders $m$.

\section{CONSTRUCTION OF THE COUPLING MATRIX}

Using the model developed in Section II, we extend the construction of a coupling matrix proposed in [1] to a UCA with a center antenna element. Let the number of outer antenna elements be at least equal to the number of relevant phase modes to account for mutual coupling, i.e. $N-1=$ $2 M+1=\left\lceil 8 \frac{d}{\lambda}+9\right\rceil$. When all signals arrive under the same elevation angle $\theta$ and all antenna ports of the UCA are terminated by $Z_{0}$, the voltage over the loads is given by $\mathbf{V}_{Z_{0}}(\theta, \phi)=Z_{0}(\overline{\bar{Z}}+$ $\left.Z_{0} \overline{\bar{I}}\right)^{-1} \cdot \mathbf{V}_{0}(\theta, \phi)$ with $\mathbf{V}_{0}(\theta, \phi)=\left[V_{0,1}(\theta, \phi), V_{0,2}(\theta, \phi), \ldots, V_{0, N}(\theta, \phi)\right]^{T}$. The $2 M+1$ lowestorder phase-mode voltages of the terminated outer antenna elements are given by $V_{Z_{0}, 2, m}^{\phi}(\theta)=$ $\frac{Z_{0}}{Z_{\text {outer }, m}+Z_{0}} V_{0,2, m}^{\phi}(\theta)$, for $m= \pm 1, \ldots, \pm M$. For a fixed elevation angle $\theta$, the open-circuit phase-mode voltages are complex numbers, independent of the azimuth angle $\phi$. For the phasemode contribution of order 0, we derive from Fig. 1:

$$
\left(\begin{array}{c}
V_{Z_{0}, 1,0}^{\phi}(\theta) \\
V_{Z_{0}, 2,0}^{\phi}(\theta)
\end{array}\right)=Z_{0}\left(\begin{array}{cc}
Z_{0}+Z_{1,1} & (N-1) Z_{1,2} \\
Z_{1,2} & Z_{0}+Z_{\text {outer }, 0}^{\phi}
\end{array}\right)^{-1} \cdot\left(\begin{array}{c}
V_{0,1,0}^{\phi}(\theta) \\
V_{0,2,0}^{\phi}(\theta)
\end{array}\right) .
$$

Consider now an ideal uniform circular array with center element consisting of stand-alone dipole elements without mutual coupling, described by phase-mode voltages $V_{0,1, m}^{\phi,(N C)}(\theta)$, for the center element, and $V_{0,2, m}^{\phi,(N C)}(\theta)$, for the outer elements. As both the realistic and the ideal array have about the same size, the number of relevant phase modes is identical, only their relative contribution differs. For the outer antennas, each phase-mode contribution of order $m= \pm 1, \ldots, \pm M$ is compensated by introducing a phase-sequence impedance $Z_{\mathrm{outer}, m}^{\phi,(M C)}$ that maps the ideal phasemode voltage onto the phase-mode voltage of an outer element in the real array:

$$
V_{Z_{0}, 2, m}^{\phi}(\theta)=\frac{Z_{0}}{Z_{\text {outer }, m}^{\phi}+Z_{0}} V_{0,2, m}^{\phi}(\theta)=\frac{Z_{0}}{Z_{\text {outer }, m}^{\phi,(M C)}+Z_{0}} V_{0,2, m}^{\phi,(N C)}(\theta) .
$$

We match the zero-order phase-modes using a phase-sequence impedance $Z_{1,1}^{(M C)}$, for the center antenna, and $Z_{\text {outer,0 }}^{\phi,(M C)}$, for the outer antennas, by imposing $V_{Z_{0}, 1,0}^{\phi}(\theta)=\frac{Z_{0}}{Z_{1,1}^{(M C)}+Z_{0}} V_{0,1,0}^{\phi,(N C)}(\theta)$ 
and $V_{Z_{0}, 2,0}^{\phi}(\theta)=\frac{Z_{0}}{Z_{\text {outer }, 0}^{\phi,(M C}+Z_{0}} V_{0,2,0}^{\phi,(N C)}(\theta)$, and by relying on (1). For a UCA with one center element and $N-1$ outer elements, $N$ mode components are compensated, i.e. order 0 for the inner element ( 1 mode component) and for the outer elements $N-1$ components of orders $M=$ $-\frac{N-2}{2}, \ldots, \frac{N-2}{2}$ when $N-1$ is odd, or $M=-\frac{N-3}{2}, \ldots, \frac{N-1}{2}$ when $N-1$ is even. Finally, one obtains for the coupling matrix $\overline{\bar{C}}$ :

$$
\mathbf{V}_{Z_{0}}(\theta, \phi)=\overline{\bar{C}} \cdot \mathbf{V}_{Z_{0}}^{(N C)}(\theta, \phi)=Z_{0}\left(\overline{\bar{Z}}_{M C}+Z_{0} \overline{\bar{I}}\right)^{-1} \cdot \mathbf{V}_{Z_{0}}^{(N C)}(\theta, \phi),
$$

where the compensating impedance matrix for the UCA with center element $\overline{\bar{Z}}_{M C}$ is given by

$$
\overline{\bar{Z}}_{M C}=\left(\begin{array}{c|c}
Z_{1,1}^{M C} & \mathbf{0}_{1 \times(N-1)} \\
\hline \mathbf{0}_{(N-1) \times 1} & \overline{\bar{Z}}_{\text {outer }}^{M C}
\end{array}\right)
$$

\section{EXAMPLE}

In Fig. 2 a dual-band dipole antenna, consisting of a dipole with length $l=15.23 \mathrm{~cm}$ surrounded by two parasitic radiators at a distance $d=1.30 \mathrm{~cm}$ and of length $l_{p}=6.65 \mathrm{~cm}$, was tuned to $50 \Omega$ at $900 \mathrm{MHz}$ and $1800 \mathrm{MHz}\left(S_{11}<-10 \mathrm{~dB}\right)$, in order to serve as outer antenna element. The nine outer antenna feeds are distributed uniformly on a circle of radius $r=8.06 \mathrm{~cm}$. As for the center element, we consider a dipole with length $l=15.35 \mathrm{~cm}$, surrounded by nine parasitic radiators at a distance $d=1.32 \mathrm{~cm}$ and of length $l_{p}=5.86 \mathrm{~cm}$. All antenna terminals are loaded by an impedance $Z_{0}=50 \Omega$. In this type of array configuration, mutual coupling shall be severe, as currents also flow on the parasitic wires that ensure dual band behavior and since several antenna wires shadow the radiation from the other antennas. Yet, for the outer elements and at $900 \mathrm{MHz}$ only $N-1=$ $2 M+1=9$ relevant phase-modes $\left|V_{Z_{0}, 2, m}^{\phi}\left(75^{\circ}\right)\right|,|m| \leq 4$, describe the mutual coupling. For the center antenna element, one single phase mode component $V_{Z_{0}, 1,0}^{\phi}\left(75^{\circ}\right)$ accounts for mutual coupling. In this case the coupling matrix fully compensates mutual coupling. At $1800 \mathrm{MHz}$, not all the relevant phase-mode voltage components describing the outer antenne elements are located in the region $|m| \leq 4$ but extend to $|m| \leq 6$, as indicated by the black bars in Fig. 3. In that case, the coupling matrix cannot fully compensate for mutual coupling effects: The grey bars in 3 show that for $N-1=9$ one can only match phase modes of order $|m| \leq 4$. Note that the white bars in 3 show the phase-modes $V_{0,2, m}^{\phi,(N C)}\left(75^{\circ}\right)$ of a stand-alone $\frac{\lambda}{2}$ dipole antenna at $1800 \mathrm{MHz}$, used as an outer element in the ideal UCA without mutual coupling.

Consider three uncorrelated sources emitting 10000 bit pseudo-random bit sequences. All signals are incident along a fixed elevation angle $\theta=75^{\circ}$, with directions of arrival $\phi_{1}=55^{\circ}, \phi_{2}=95^{\circ}$ and $\phi_{3}=143^{\circ}$. The three signals are received in the presence of additive white Gaussian noise. Using the compensated MUSIC algorithm, in Table I the mean DOA estimates and the standard deviation are presented at $900 \mathrm{MHz}$ and $1800 \mathrm{MHz}$, for an ensemble of 500 implementations, for equally strong signals and for an $\mathrm{SNR}=3 \mathrm{~dB}$. At $900 \mathrm{MHz}$, all mutual coupling effects are fully taken into account by this coupling matrix. At $1800 \mathrm{MHz}$, a systematic error is found in the mean DOA estimates, as the coupling matrix does not compensate the phase modes of orders $m= \pm 5$ and $m= \pm 6$ (Fig. 3), yet these components are relevant to the EM behavior of the outer antenna elements in the UCA. However, by increasing the number of outer antenna elements to $N-1=13$ (14 antenna elements in total), the coupling matrix compensates phase modes of orders $|m| \leq 6$ and the MUSIC algorithm based on the coupling matrix yields very accurate results at $1800 \mathrm{MHz}$.

\begin{tabular}{|c|r|r|r|}
\hline & \multicolumn{1}{|c|}{$f=900 \mathrm{Mhz}$} & \multicolumn{1}{|c|}{$f=1800 \mathrm{Mhz}$} \\
\hline N-1 & 9 & \multicolumn{1}{c|}{9} \\
\hline DOA 1 & $55.03^{\circ}\left(0.50^{\circ}\right)$ & $54.95^{\circ}\left(0.10^{\circ}\right)$ & $55.00^{\circ}\left(0.06^{\circ}\right)$ \\
DOA 2 & $94.97^{\circ}\left(0.77^{\circ}\right)$ & $94.79^{\circ}\left(0.11^{\circ}\right)$ & $95.00^{\circ}\left(0.06^{\circ}\right)$ \\
DOA 3 & $142.95^{\circ}\left(0.42^{\circ}\right)$ & $143.12^{\circ}\left(0.10^{\circ}\right)$ & $143.00^{\circ}\left(0.05^{\circ}\right)$ \\
\hline \multicolumn{3}{|c}{ TABLE I }
\end{tabular}

MEAN AND STANDARD DEVIATION FOR DOA ESTIMATION BASED ON MUSIC WITH COUPLING MATRIX. 


\section{REFERENCES}

[1] H. Rogier and E. Bonek, "Analytical spherical-mode based compensation of mutual coupling in uniform circular arrays for direction-of-arrival estimation," Int. Journal of Electr. and Comm., p. in press, 2005.

[2] B. Schaer, K. Rambabu, J. Bornemann, and R. Vahldieck, "Design of reactive parasitic elements in electronic beam steering arrays," IEEE Trans. Antennas Propag., vol. 53, pp. 1998-2003, Jun. 2005.

[3] E. Taillefer, A. Hirata, and T. Ohira, "Direction-of-arrival estimation using radiation power pattern with an ESPAR antenna," IEEE Trans. Antennas Propag., vol. 53, pp. 678-684, Feb. 2005.

[4] H. Rogier, "Phase-mode based construction of a coupling matrix for uniform circular arrays with a center element," Microwave Opt. Technol. Lett., p. accepted for publication, 2005.

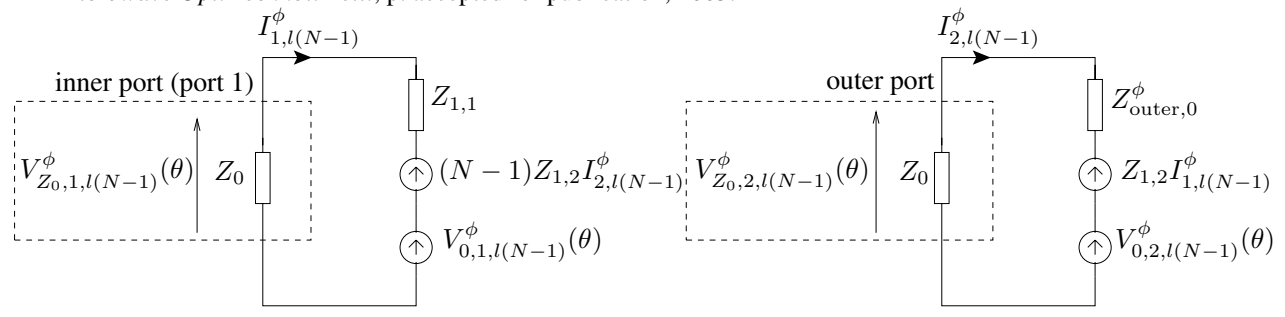

Fig. 1. Circuit model of the UCA in receive mode, for phase modes of the orders $l(N-1)(l=0,1, \ldots)$.

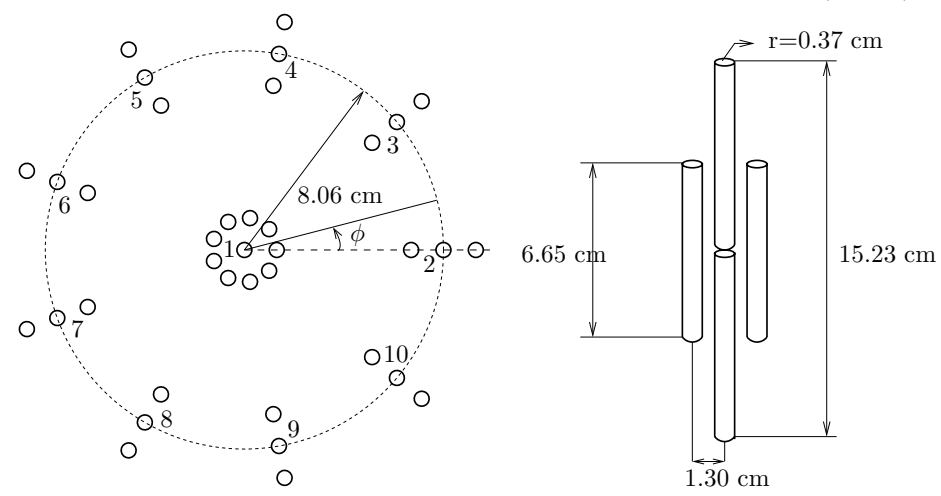

nine-element UCA constellation with center element

outer dualband dipole $(2-9)$

Fig. 2. UCA with center element, consisting of nine outer dual-band dipole antennas and center dual-band dipole antenna.

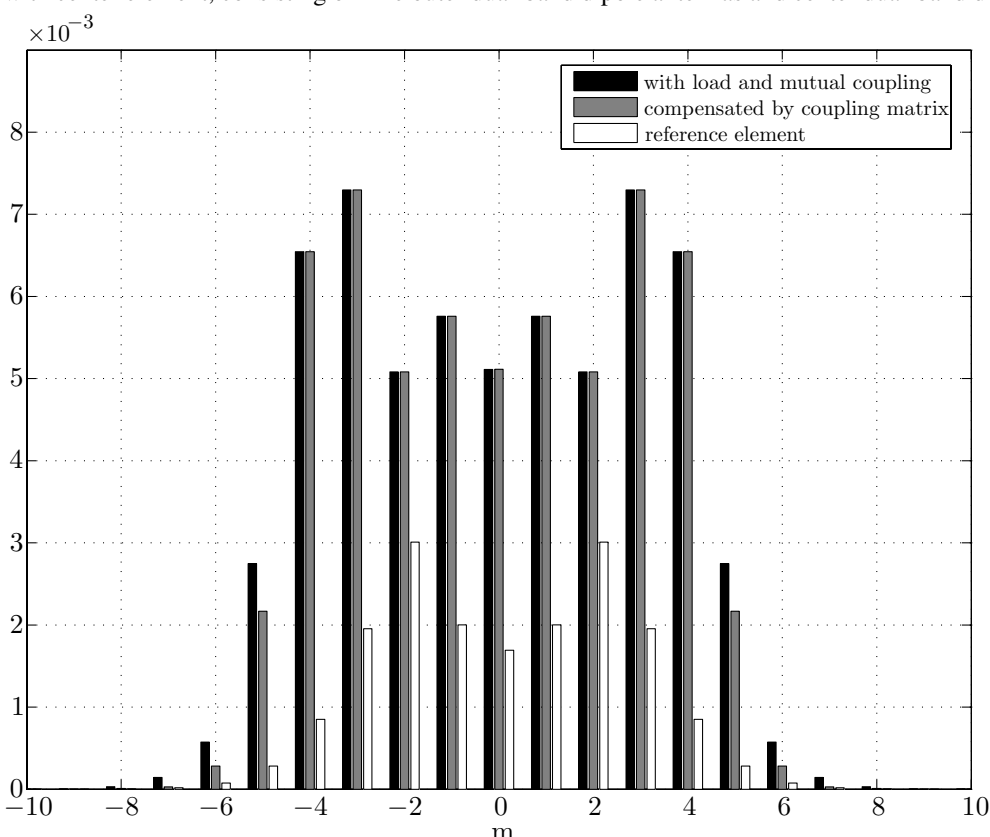

Fig. 3. Phase-mode voltages $V_{Z_{0}, 2, m}^{\phi}\left(75^{\circ}\right)$ at $1800 \mathrm{MHz}$ for the outer elements of the UCA with center element. 


\section{IEEE Antennas and Propagation Society International Symposium 2006}
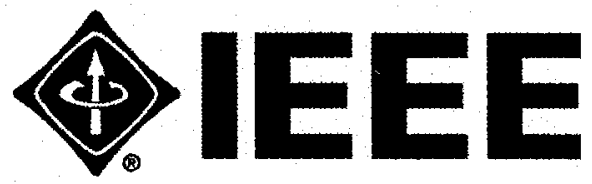

Copyright and Reprint Permissions: Abstracting is permitted with credit to the source. Libraries are permitted to photocopy beyond the limit of U.S. copyright law for private use of patrons those articles in this volume that carry a code at the bottom of the first page, provided the per-copy fee indicated in thecode is paid through Copyright Clearance Center, 222 Rosewood Drive, Danvers, MA 01923.

For other copying, reprint or republications permission, write to IEEE Copyrights Manager, IEEE Operations Center, 445 Hoes Lane, Piscataway, New Jersey USA 08854. All rights reserved.

IEEE Catalog Number:

ISBN:

Library of Congress No.:
$06 \mathrm{CH} 37758 \mathrm{C}$

1-4244-0123-2

$90-640397$ 


\title{
2006 IEEE Antennas and Propagation
} Society International Symposium

\section{with \\ USNC/URSI National Radio Science and AMEREM Meetings}

\section{Symposium Digest Volume 2 of 5}

\author{
9-14 July 2006 \\ Albuquerque Convention Center \\ Hyatt Regency Hotel
Albuquerque, NM USA

\section{and Hyatt Regency Hotel
Albuquerque, NM USA}

9 IEEE Catalog \#: 06CH37758 ISBN: 1-4244-0122-4 Library of Congress: 90-640397

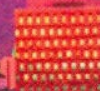

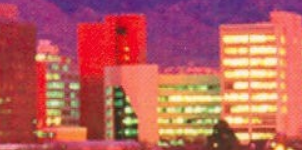

19.

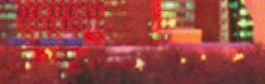
कor .
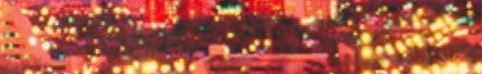